\title{
Analysis of Functional Relationships between Rice Particles and Oral Perception Using Amazake: A Traditional Japanese Beverage of Malted Rice
}

\author{
M. Ajiro, M. Araki, M. Ishikawa, K. Kobayashi, I. Ashida*, Y. Miyaoka \\ Department of Health and Nutrition, Niigata University of Health and Welfare, Niigata, Japan \\ Email: *asida@nuhw.ac.jp
}

How to cite this paper: Ajiro, M., Araki, M., Ishikawa, M., Kobayashi, K., Ashida, I. and Miyaoka, Y. (2017) Analysis of Functional Relationships between Rice Particles and Oral Perception Using Amazake: A Traditional Japanese Beverage of Malted Rice. Food and Nutrition Sciences, 8, 901-911. https://doi.org/10.4236/fns.2017.810065

Received: September 9, 2017

Accepted: October 9, 2017

Published: October 12, 2017

Copyright $\odot 2017$ by authors and Scientific Research Publishing Inc. This work is licensed under the Creative Commons Attribution International License (CC BY 4.0).

http://creativecommons.org/licenses/by/4.0/

c) (i) Open Access

\begin{abstract}
The present study aimed to examine the effects of rice particles in test foods and their visual appearance on oral textural perceptions. One original and three filtered Amazake (a traditional Japanese beverage made from malted rice) preparations were used as test foods. Three physical measurements of the test foods were conducted at room temperature: linear spread test, viscosity, and concentration of rice particles. Results of these three measurements formed two groups with lesser and more filtered test foods. Sensory evaluation experiments using a paired comparison test in 32 healthy young participants revealed the following: 1) the estimates of "grittiness in the mouth" (mouth feel) and "grittiness at a glance" of the test foods also comprised two groups, similar to the results of the physical measurements, 2) estimates of textural "smoothness" resulted in two groups, with a half-range of two items of "grittiness", and 3) estimates of "sweetness" and "odor intensity" were similar to each other than to the other three items. Functional relationships between physical properties and characteristics of the test foods and sensory evaluation of their grittiness (oral, textural, and visual) of Amazake are discussed.
\end{abstract}

\section{Keywords}

Amazake, Rice Particles, Grittiness, Oral Textural Perception, Human

\section{Introduction}

A keystone study classified textural parameters of foods and proposed three main categories [1]. Of these three categories, one class encompassing geome- 
trical characteristics was associated with grittiness, smoothness, and other textural properties of food. These geometrical characteristics appear to be less understood than mechanical characteristics, which is another class including characteristics such as hardness, cohesiveness, and adhesiveness. This maybe because geometrical characteristics are more complicated and involve additional factors. A subsequent study modified and reconfigured textural parameters and proposed a texture profile [2]. One important characteristic of the profile is that texture profiles should include "visual appearance" of foods, because vision is a potent sensory system that can lead to textural and flavor expectations regarding food [3]. For example, a study showed that visual input affected the perceived oral texture of vanilla custard dessert [4].

Amazake is a Japanese food produced from malted rice or sake lees. Because both types of Amazake are nutritious and flavorsome, they are recommended to people of all age groups. However, the presence of rice particles owing to malted rice apparently renders people hesitant to consume it [5]. In general, suspended particles, including malted rice in Amazake, can elicit distinct oral perceptions including grittiness, smoothness, and roughness [6]. Artificial, uniform, and small $(<1 \mathrm{~mm}$ diameter) particles are generally used in experiments for the examination of oral perceptions; however, rice particles in Amazake are natural products and are neither uniform nor small.

To the best of our knowledge, few studies have examined oral perceptions using natural particle products in suspension. In the present study, we examined the effects of particles in Amazake test foods prepared using malted rice and visual observation of the test foods on oral textural perceptions. The results showed that the concentration of particles did not simply reflect grittiness or smoothness, and that visual observation strongly affected these perceptions.

\section{Materials and Methods}

\subsection{Test Foods and Physical Measurements}

\subsubsection{Test Foods}

A commercially available bottled Amazake (Houraiya Ltd., Niigata, Japan) was used as basic test food in the present study. The test food was filtered (under gravity) using three different sieves of pore diameters $1.5,3.0$, and $5.0 \mathrm{~mm}$ (Table 1). Four test foods, three filtered and the original non-filtered, were used in experiments. For convenience, these test foods were numbered \#1 to \#4 according to the quantity of particles. Food \#1 was filtered through the smallest 1.5-mm diameter filter, while test food \#4 was not filtered (Figure 1(a)). Figure 1 (b) depicts one of the rice particles in the test foods. Table 2 shows the average size of the rice particles in the four test foods. Weight of suspended particles in the three filtered test foods was approximately $80 \%$ (\#3), 50\% (\#2), and 40\% (\#1) of the test reference food \#4 (Table 1). These percentages describe the weight of suspended particles in the food. For example, in test food $\# 3,80 \%$ of the original test food comprised suspended particles, and $20 \%$ comprised the filtrate. 
Table 1. Amazake solutions as test foods.

\begin{tabular}{ccccc}
\hline Test food & $\# 1$ & $\# 2$ & $\# 3$ & $\# 4$ \\
\hline Pore diameter $(\mathrm{mm})$ & 1.5 & 3.0 & 5.0 & $-^{\mathrm{a}}$ \\
Weight after filtration $(\mathrm{g})$ & 3.8 & 5.1 & 8.9 & 10.0 \\
& $(0.3)$ & $(0.7)$ & $(0.5)$ & $(0.0)$ \\
\hline
\end{tabular}

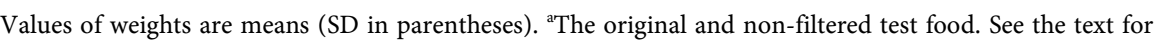
details.

Table 2. Average size of rice particles in the test foods.

\begin{tabular}{cccc}
\hline & Length & Width & Height \\
\hline Size $(\mathrm{mm})$ & 3.3 & 2.3 & 1.6 \\
& $(0.87)$ & $(0.47)$ & $(0.35)$ \\
\hline
\end{tabular}

Values of dimensions are means (SD in parentheses).

(a)

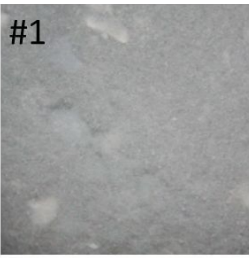

\#3

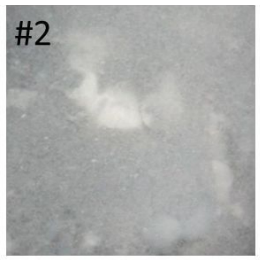

\#4 (b)

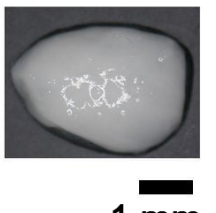

$1 \mathrm{~mm}$

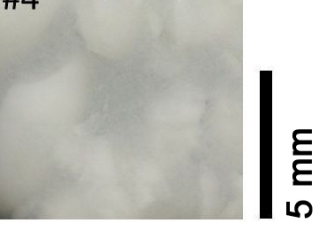

Figure 1. Photomicroscopy of the test foods. (a) External appearance of the four test foods used (See the text for details of \#1,\#2,\#3, and \#4); (b) A rice particle in the test foods.

The Brix value and $\mathrm{pH}$ of the test foods were $21.3 \%(\mathrm{n}=5)$ and $5.2(\mathrm{n}=5)$, respectively. The overall intensity of odor of the four test foods was measured mechanically (XP-329III, New Cosmos Electric CO., LTD, Osaka, Japan). One-way analysis of variance (ANOVA) of the measured values revealed that average intensities were not statistically different among the four test foods.

\subsubsection{Physical Measurements}

Three physical measurements of the four test foods were conducted at room temperature (i.e., $25^{\circ} \mathrm{C}$ ) as reference for the results of sensory evaluation. First, linear spread test [7], Saraya Co., Ltd., Osaka, Japan) values were measured ( $\mathrm{n}=$ 5) to assess fluidity of the test foods. A stainless steel ring with an inner diameter of $30 \mathrm{~mm}$ was set at the center of concentric circles and was filled with $20 \mathrm{~mL}$ of the test sample. The ring was vertically lifted and gently removed, and the distance from the center of the sample spread was measured at six points. Consistency of the sample was objectively measured as the dimensions of sample spread in diameter. The term "fluidity", which is the reciprocal of dynamic vis- 
cosity [8], was used as an index of "smoothness" in the present study because both "viscosity" and "smoothness" were considered to be closely associated with each other, because they create "creaminess" in the oral cavity [9].

Second, the viscosity of test foods was determined using a single cylinder rotational viscometer (TVB-10M, TOKI SANGYO CO., LTD, Tokyo, Japan). A 500 $\mathrm{mL}$ glass beaker (inner diameter $85 \mathrm{~mm}$ ) was filled with one of the test foods and placed under a measurement rotor with a diameter of $25 \mathrm{~mm}$. The rotor was selected for lower viscosity samples, such as the present test foods, and was vertically immersed in the test food three times. Rotational speed and time were determined according to the manufacturer's instructions: 1) rotational speed of 20 rpm was determined as the lowest speed that could avoid both over- and under-scale errors due to excessive over- and under-torquing, and 2) the time point $60 \mathrm{~s}$ after the start of measurements was determined as the minimum time at which measurement values were stabilized. Rotational viscosity was measured as stress applied to the rotor (measured in $\mathrm{mPa} \cdot \mathrm{s}$ ).

Finally, the concentration of rice particles was determined: 1) $7 \mathrm{~g}$ of each filtered test food was leveled off using a spoon, 2) the sample was spread evenly on a paper sheet, and 3) number of clear (i.e., countable) rice particles (see Figure 1(b)) was counted five times by visual observation to calculate the concentration.

\subsection{Sensory Evaluation Experiment}

\subsubsection{Participants}

Thirty-two healthy young adults [16 males and 16 females, $20.5 \pm 2.9$ (mean \pm SD) years old] participated in this study. The participants received remuneration and were undergraduate students of Niigata University of Health and Welfare, and were not trained for sensory evaluations in this study. None of the participants had any subjective problems with gustatory and olfactory functions. Informed consent was obtained from all participants.

\subsubsection{Procedures}

The experiment was conducted in an air-conditioned room maintained at $25^{\circ} \mathrm{C}$. An experimental set consisted of eight participants simultaneously, with four such sets in total. Participants were comfortably seated on chairs. After instructions were delivered by the experimenter, participants were invited to ask any questions about the experiment. During the experiment, participants were required not to talk to the others concerning any matters related to the experiment. Subsequently, participants were asked to rinse their mouths with tap water and were instructed to repeat rinsing whenever the test foods were changed. A session consisted of $12[4 \times(4-1)]$ trials for each participant, with a 1-min break between trials. A 5-min rest period was also introduced after the first half of each session to prevent participant fatigue.

A modified method of Scheffe's paired comparison was used. Each participant was provided with two foods $(10 \mathrm{~mL}$ each), which were randomly selected in 
each trial, and they were asked to compare the perceived intensity of the latter with that of the former. Five items were adopted for evaluation: 1) "grittiness at a glance", 2) "overall intensity of the odor", 3) "grittiness in the mouth", 4) "smoothness", and 5) "sweetness." The former two items were evaluated before ingestion and the latter three items after ingestion. The comparison of intensity was quantified on a seven-step graded scale (from -3 to +3 ).

\subsection{Statistical Analysis}

After Bartlett's test for examining homogeneity of variances, physical measurements were analyzed using one-way ANOVA for parametric variables (linear spread test [LST] and viscosity) and using Kruskal-Wallis test for non-parametric variables (the concentration of rice particles). Confidence intervals between pairs of test foods were estimated to detect specific differences. Scores obtained from sensory evaluation were statistically analyzed using three-way ANOVA using "main effect (the five items)", "participant", and "main effect $\times$ participant", based on the design of the modified Scheffe's method. Differences were regarded as statistically significant where $P<0.05$.

\section{Results}

\subsection{Physical Measurement}

As seen in Figure 2(a), the average LST values for the four test foods decreased from test food \#1 to \#4. The average percentage decreases in LST values were $0.5 \%$ from test foods \#1 to \#2 and 5.6\% from test foods \#3 to \#4, whereas the average percentage decrease was $12.4 \%$ from test foods $\# 2$ to $\# 3$, suggesting that there are two LST groups separating test foods \#2 and \#3. Average viscosity values increased from test foods \#1 to \#4 (Figure 2(b)). The viscosity values of \#1 and $\# 2$ were clearly far less than those of $\# 3$ and $\# 4$. The percentage increase in viscosity values from test foods \#2 to \#3 exceeded $3800 \%$ (Figure 2(b)). Statistical analysis revealed significant differences in LST and viscosity values among
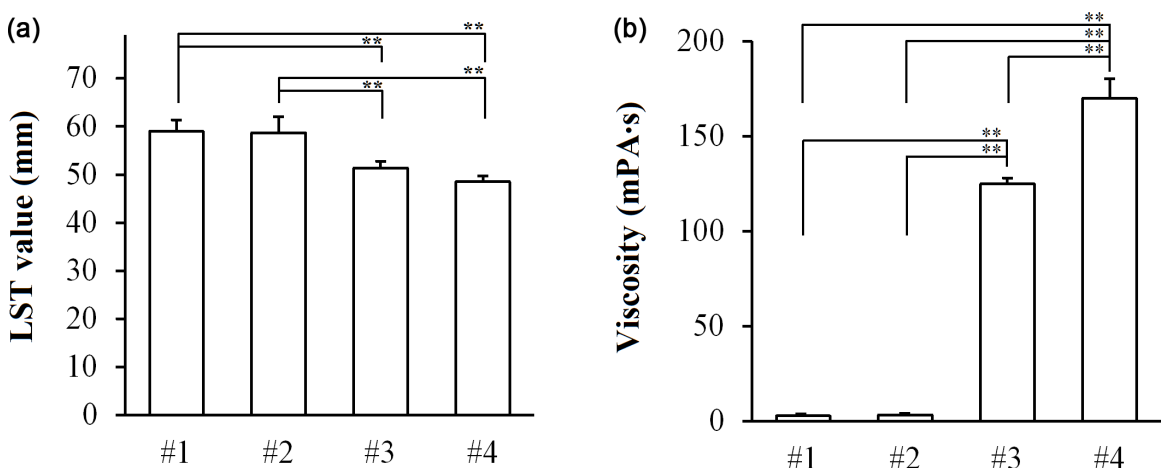

Figure 2. Average linear spread test (LST) and viscosity values of the test foods. (a) Average LST values using an LST set (see the text for details); (b) Average viscosity values measured using a rotatory viscometer. Bars and error lines indicate the means and standard deviations of the measurements. Horizontal lines with asterisks indicate specific differences in these two values between pairs of test foods $\left({ }^{\star *} P<0.01 ;{ }^{\star} P<0.05\right)$. 


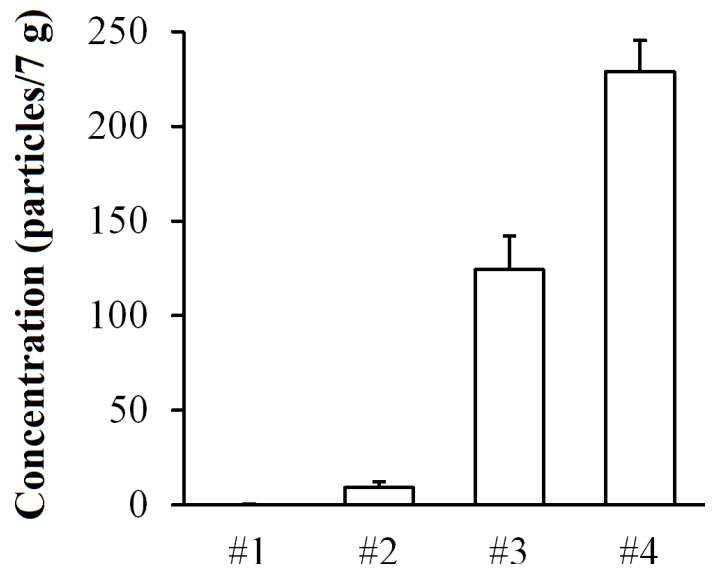

Figure 3. Concentration of rice particles in the test foods. Number of particles per $7 \mathrm{~g}$ of test foods was determined by visual observation (see the text for details). Bars and error lines indicate the means and standard deviations of measurements. Horizontal lines with asterisks indicate specific differences in concentrations between pairs of the test foods $\left({ }^{* *} P<0.01\right)$.

the four test foods, and specific differences were also identified between four of the 12 possible pairs of test foods for LST values (Figure 2(a)) and between five pairs of viscosity values (Figure 2(b); $P<0.05$ ).

Figure 3 shows the average concentration of rice particles found in the four test foods. The concentration increased from test foods \#1 (with no visible rice particles) to \#4. Percentage changes in the concentrations were $13.5 \%$ (for \#3) and $24.9 \%$ (for \#4) of \#2 test foods, respectively. There were significant differences in concentrations among test foods except for test food \#1 $(P<0.01)$, and specific differences were also detected between all three pairs of the test foods $\left(P_{\mathrm{s}}<0.05\right)$.

\subsection{Sensory Evaluation}

Figure 4 depicts sensory evaluation estimates of the five items for the four test foods used in the present study. The estimates of "grittiness at a glance" (Figure 4(a)), "grittiness in the mouth (Figure 4(b))", and "smoothness" (Figure 4(c)) decreased as the amount of rice particles diminished as a result of increasing residue on the sieves (Table 1). These three sets of estimates clearly divided into two groups, with two test foods each (\#1 and \#2; \#3 and \#4), although the range between the two groups in the estimates of "smoothness (Figure 4(c))" was approximately half of the two "grittiness" measures (Figure 4(a) and Figure 4(b)). Ranges between these estimates in "sweetness" (Figure 4(d)) and "odor intensity" (Figure 4(e)) are much smaller than those for the other three items (Figures $4(a)-(c))$. Statistical analysis (by the modified Scheffe's method) detected significant differences in all the main effects (Figures 4(a)-(e); $P_{S}<0.01$ ). Numbers of specific differences between the estimates were four (Figure 4(a)), five (Figure 4(b)), four (Figure 4(c)), two (Figure 4(d)), and three (Figure 4(e)) of the 12 possible pairs of the test foods $\left(P_{\mathbf{S}}<0.05\right)$. 
(a) Gritteness in the mouth

(b) Gritteness by look

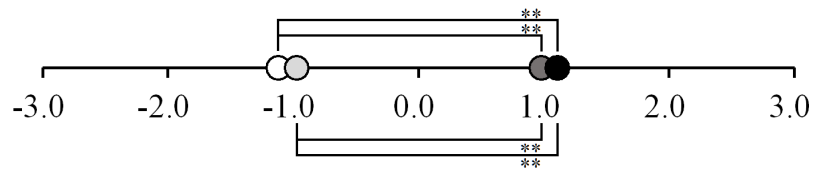

(c) Smoothness

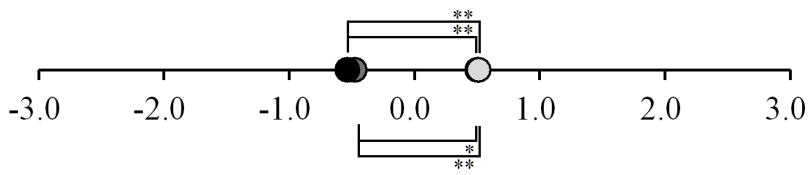

(d) Sweetness

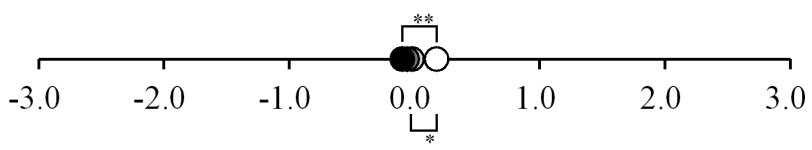

\section{(e) Odor intensity}

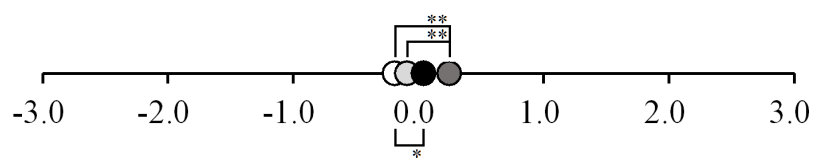

Figure 4. Estimates for perceived intensities of test foods. White, light gray, dark gray, and black circles on the line indicate \#1, \#2, \#3, and \#4 test foods, respectively. Horizontal lines with asterisks indicate specific differences in estimates between pairs of the test foods $\left({ }^{* *} P<0.01 ;{ }^{*} P<0.05\right)$.

\section{Discussion}

Particle concentration is one of the major determinants of grittiness of food in the mouth [10] [11]. The concentrations of rice particles in the test foods were clearly different from each other (Figure 2), whereas estimates of "grittiness in the mouth" by the sensory evaluation experiment identified two separate groups: test foods \#1 and \#2 and test foods \#3 and \#4 (Figure 4(b)). The grouping of these estimates seems to be inconsistent with the findings of previous studies [10] [11], in which grittiness was proportional to the concentration of particles. Indeed, the concentration of particles of test foods \#1 and \#2 correspond to the estimates of grittiness for these test foods, whereas the concentration of particles of test foods \#3 and \#4 does not. It is necessary to consider two different aspects, such as food and sensation, to explain this inconsistency. In terms of the food aspect, rice particles used in the present study are largely different, not only in concentration, but also in size and type from those used in previous studies [10] [11]. The average size of rice particles in the present study (Table 2) was much greater than those in previous studies, and showed an indeterminate (Figure 1 (b)) rather than spherical shape [10] [11]. In the sensation aspect, the intensity of "grittiness" evoked by test foods \#1, \#2, and \#3 (Figure 4(b)) increased cor- 
respondingly with a progressive increase in particle concentration (Figure 3). Conversely, "grittiness" evoked by the test foods \#3 and \#4 were not statistically different, although their particle concentrations were clearly different (Figure 3). According to a classical psychophysical law (see Figure 2 in [12]), the intensity of grittiness in this study would not correspond to that of test food \#4 if the stimulus exceeded the strongest one (i.e., terminal threshold), and the intensity of grittiness might occur with a "saturated" type of sensation.

Estimates of "grittiness at a glance" (Figure 4(a)) were very similar to those of "grittiness in the mouth" (Figure 4(b)). This similarity is interesting, and the reasons for this similarity are difficult to explain. One possibility is that "grittiness at a glance" affected the perceived "grittiness in the mouth" owing to "vision-touch interactions" [3], as visual estimation of grittiness preceded the oral estimation in the present experiment. A previous study used a special food delivery cup, which consisted of two compartments; the upper compartment contained the "upper custard" and the lower compartment contained the "ingested custard" [4]. In that study, participants consumed custard dessert with larger first bites as the "upper custard" compartment was more favorable looking, despite participants ingesting the same custard from the "ingested custard" compartment. The present study suggested the significance of visual cues of food in determining bite size. The similarity between "grittiness at a glance" and "grittiness in the mouth" in our study may be a result of visual cues regarding food in oral textural perception: participants in the present study estimated "grittiness in the mouth" by visual observation prior to ingestion.

The average LST (Figure 2(a)) and viscosity values (Figure 2(b)) were consistent with estimates of "smoothness" (Figure 4(c)) as lower LST and higher viscosity values suggest greater consistency of fluid foods. A previous study showed that LST values enabled the separation of fluid foods for dysphagic patients into two categories but were not predictive of viscosity [7]. In the present study, both LST values and estimates of "smoothness" revealed two groups (test foods \#1 and \#2 and test foods \#3 and \#4), similar to the two divisions of "grittiness." Furthermore, there were no significant differences between the groups in terms of LST values and estimates of "smoothness", whereas the viscosity of test food \#3 differed significantly from that of test food \#4 (Figure 2(b)). Thus, "smoothness" is likely to be more closely associated with LST values than measures of viscosity.

Estimates of the two chemical sensory items, the overall intensity of odor and sweetness, of the test foods were much more similar to each other (Figure 4(d) and Figure 4(e)) than to the other three categories (Figures 4(a)-(c)). However, estimates of the two chemical sensory items showed, in part, statistically significant differences, in spite of the fact that these chemical components should be identical among the four test foods (see "2.1.1 Test foods" in "Materials and Methods"). An excellent review documented important roles of vision in foodrelated behaviors [3] and identified that "vision is strongly involved in food dis- 
crimination and selection and can lead flavor and texture expectations about a food." Unfortunately, the authors cited studies related to color vision only. The present results (Figure 4(d) and Figure 4(e)) may involve examples that demonstrate the influence of visual information of food on sensory evaluations of smell and taste. However, it is unclear which factors pertaining to visual information may be effective: shape, size, texture, and so on. Another possible explanation for significant differences in estimates of "sweetness" is that "grittiness in the mouth" influences sweetness of the test foods, as oral texture can modify the sensitivity of sweetness [13] and viscosity can modify not only sensitivity [14] [15] but also intensity [16] [17] of the four basic tastes. For example, it is well known that viscosity affects sweetness perception in beverages, i.e., increasing viscosity results in a reduced intensity of sweetness [18]. Although the results of the present study also supported this finding, the reason for the small decrease in perceived sweetness intensity was untrained participants.

The first major limitation of the present study is associated with the test foods used. Three of the four test foods were filtered using three different sieves (Table 1 ), but their pore sizes may have been inappropriate considering the size of the resulting rice particles (Table 2). The average particle diameter $(3.3 \mathrm{~mm}$ ) was slightly greater than the pore size of the second largest sieve $(3.0 \mathrm{~mm})$ and considerably smaller than that of the largest sieve $(5.0 \mathrm{~mm})$. The large difference in pore size between these two sieves is probably responsible for the large difference in concentrations between test foods \#2 and \#3 (Figure 3). However, the difference in concentrations did not result in large differences in the number of test foods that exhibited a stepwise increase from test foods \#1 to \#4 (Table 1). This limitation suggests that a sieve of approximately $4.0 \mathrm{~mm}$ diameter may be better suited to the filtration of rice particles to prevent a gradient in their concentration if similar experiments on Amazake are conducted in the future. The second major limitation was that two properties of the rice particles, concentration and size, have been contaminated among the four test foods, and they cannot be disentangled in this study. It would be necessary to compare perceived intensity of the test food at the same concentration with different particle sizes to arrive at a clear conclusion. The final limitation is that there were numerous small particles in the test foods and the present study did not consider these. Some of the smaller particles were half the size of particles shown in Table 2, and others were even smaller and undetectable by visual observation. Influences of these smaller particles on visual and oral perception of Amazake are unknown, but previous studies have suggested potential roles of such particles on sensory perception [6] [10] [11].

\section{Conclusion}

The concentration of Amazake's particles does not simply reflect oral grittiness or smoothness, and visual observation of the particles strongly affects these perceptions. 


\section{Acknowledgements}

The authors would like to thank Enago (https://www.enago.jp/) for the English language review.

\section{References}

[1] Szczesniak, A.S. (1963) Classification of Texture Characteristics. Journal of Food Science, 28, 385-389. https://doi.org/10.1111/j.1365-2621.1963.tb00215.x

[2] Sherman, P. (1969) A Texture Profile of Foodstuffs Based Upon Well-Defined Rheological Properties. Journal of Food Science, 34, 458-462. https://doi.org/10.1111/j.1365-2621.1969.tb12804.x

[3] Verhagen, J.V. and Engelen, L. (2006) The Neurocognitive Bases of Human Multimodal Food Perception: Sensory Integration. Neuroscience and Biobehavioral Reviews, 30, 613-650. https://doi.org/10.1016/j.neubiorev.2005.11.003

[4] de Wijk, R.A., Polet, I.A., Engelen, L., van Doorn, R.M. and Prinz, J.F. (2004) Amount of Ingested Custard Dessert as Affected by Its Color, Odor, and Texture. Physiology \& Behavior, 82, 397-403. https://doi.org/10.1016/j.physbeh.2004.04.053

[5] Kawazome, S. and Tanaka, T. (1974) Food Preferences of Young People (In Japanese with English Abstract). The Japanese Journal of Nutrition and Dietetics, 32, 32-38. https://doi.org/10.5264/eiyogakuzashi.32.32

[6] Engelen, L. and van der Bilt, A. (2008) Oral Physiology and Texture Perception of Semisolids. Journal of Texture Studies, 39, 83-113. https://doi.org/10.1111/j.1745-4603.2007.00132.x

[7] Nicosia, M.A. and Robbins, J. (2007) The Usefulness of the Line Spread Test as a Measure of Liquid Consistency. Dysphagia, 22, 306-311.

https://doi.org/10.1007/s00455-007-9086-3

[8] Bourne, M. (2002) Food Texture and Viscosity. Academic Press, New York. https://doi.org/10.1016/B978-012119062-0/50001-2

[9] Szczesniak, A.S. (1987) Correlating Sensory with Instrumental Texture Measurements-An Overview of Recent Developments. Journal of Texture Studies, 18, 1-15. https://doi.org/10.1111/j.1745-4603.1987.tb00566.x

[10] Engelen, L., de Wijk, R.A., van der Bilt, A., Prinz, J.F., Janssen, A.M. and Bosman, F. (2005) Relating Particles and Texture Perception. Physiology \& Behavior, 86, 111-117. https://doi.org/10.1016/j.physbeh.2005.06.022

[11] Imai, E., Hatae, K. and Shimada, A. (1995) Oral Perception of Grittiness: Effect of Particle Size and Concentration of the Dispersed Particles and the Dispersion Medium. Journal of Texture Studies, 26, 561-576. https://doi.org/10.1111/j.1745-4603.1995.tb00804.x

[12] Stevens, S.S. (1951) Mathematics, Measurement, and Psychophysics. John Wiley \& Sons, Inc., New York.

[13] Ingate, M.R. and Christensen, C.M. (1981) Perceived Textural Dimensions of Fruit-Based Beverages. Journal of Texture Studies, 12, 121-132. https://doi.org/10.1111/j.1745-4603.1981.tb01226.x

[14] Mackey, A. and Valassi, K. (1956) The Discernment of Primary Taste in the Presence of Different Food Texture. Food Technology, 10, 238-240.

[15] Stone, H. and Oliver, S.M. (1969) Measurement of the Relative Sweetness of Selected Sweeteners and Sweetener Mixtures. Journal of Food Science, 34, 215-222. https://doi.org/10.1111/j.1365-2621.1969.tb00922.x 
[16] Izutu, T., Taneya, S., Kikuchi, E. and Sone, T. (1981) Effect of Viscosity on Perceived Sweetness Intensity of Sweetened Sodium Carboxymethylcellulose Solutions. Journal of Texture Studies, 12, 259-273. https://doi.org/10.1111/j.1745-4603.1981.tb01235.x

[17] Moskowitz, H.R. and Arabie, P. (1970) Taste Intensity as a Function of Stimulus Concentration and Solvent Viscosity. Journal of Texture Studies, 1, 502-510. https://doi.org/10.1111/j.1745-4603.1970.tb00748.x

[18] Christensen, C.M. (1980) Effects of Solution Viscosity on Perceived Saltiness and Sweetness. Perception and Psychophysics, 28, 347-353.

https://doi.org/10.3758/BF03204394 